\title{
Erratum: Polarized structure of nucleon in the valon representation
}

${\text { Firooz } \text { Arash }^{a} \text { and Fatemeh Taghavi-Shahri }}^{b}$

${ }^{a}$ Physics Department, Tafresh University, Tehran Road, Tafresh, Iran

${ }^{b}$ Physics Department, Iran University of Science and Technology, University Street, Narmak, Tehran 16345, Iran

E-mail: farash@cic.aut.ac.ir, f_taghavi@iust.ac.ir

ERRATUM TO: JHEP07(2007)071

In article JHEP07(2007)071 equation (3.2) in the left hand side $\delta F_{j}(y)$ should be replaced by $\delta G_{j}$, and therefore, it reads as follows

$$
\delta G_{j}(y)=\delta F_{j}(y) G_{j}(y)=N_{j} y^{\alpha_{j}}(1-y)^{\beta_{j}}\left(1+a_{j} y^{0.5}+b_{j} y+c_{j} y^{1.5}+d_{j} y^{2}\right)
$$

